\title{
MÉTODOS DE ANÁLISE ESTRUTURAL DE TABULEIROS DE PONTES EM VIGAS MÚLTIPLAS DE CONCRETO PROTENDIDO
}

\author{
Eduardo Valeriano Alves ${ }^{1}$ \\ Sérgio Marques Ferreira de Almeida ${ }^{1}$ \\ Flávia Moll de Souza Judice ${ }^{1}$
}

Resumo: Este trabalho visa apresentar os métodos tradicionalmente empregados para análise estrutural de tabuleiros de vigas múltiplas de concreto protendido. Apresenta-se também uma síntese dos fundamentos teóricos do método dos elementos finitos, necessário à automatização da análise. Após a descrição dos métodos é feita uma avaliação das limitações dos mesmos indicando-se o conjunto de recursos necessários para que se alcançe um alto grau de automatização da análise do tipo de obras aqui tratadas.

Palavras-chave: Pontes; Análise Estrutural.

Abstract: This work presents the main processes traditionally used to analyze deck bridges made with prestressed beams. It is also presented a synthesis of the theorical fundaments of the finite element method, which is necessary for computational analysis. After the description of the processes, an evaluation of its limits is done, indicating a group of means needed to reach a high grade automation of the analysis in constructions treated in this work.

Key words: bridges, structural analysis.

\footnotetext{
${ }^{1}$ Universidade Federal Fluminense
} 


\section{INTRODUÇÃO}

A utilização de tabuleiros de vigas múltiplas de concreto protendido em pontes, elevados e viadutos é extremamente difundida no Brasil em função das vantagens econômicas e construtivas desta solução. A análise estrutural deste tipo de obra é efetuada usualmente em duas etapas. Na primeira delas, desenvolve-se a análise da superestrutura separando-a dos demais elementos integrantes do conjunto estrutural: meso e infraestrutura.

$\mathrm{Na}$ etapa de análise da superestrutura faz-se, em geral, nova simplificação. Assimila-se o modelo estrutural da grelha formada por longarinas e transversinas a um modelo menos rigoroso, representado por vigas biapoiadas. Para que esta assimilação seja feita, aplicam-se métodos tradicionais, por meio dos quais são determinadas as parcelas de carregamento correspondentes à cada uma das longarinas. Este trabalho tem como finalidade apresentar os fundamentos teóricos destes métodos e concluir apontando suas principais limitações para efeito de automatização da análise.

\section{MÉTODOS PARA ANÁLISE SIMPLIFICADA}

Na obtenção de solicitações e reações de apoio em tabuleiros de vigas múltiplas, são utilizados tradicionalmente quatro métodos aproximados de cálculo. As descrições sucintas destes métodos são apresentadas nos itens seguintes, após uma sinopse dos estudos iniciais que os precederam.

\subsection{SÍNTESE DA EVOLUÇÃO DOS MÉTODOS APROXIMADOS [1]}

Em função da sua elevada hiperestaticidade, a análise do comportamento estrutural de grelhas constituiu-se no passado em uma tarefa complexa para os projetistas. Isto motivou o desenvolvimento de diversos processos simplificados de cálculo manual.

Em 1893, Zschetzsche, com base no método das forças, desenvolveu um trabalho, no qual não obteve êxitos maiores em aplicações práticas, face às dificuldades e complexidades numéricas de cálculo [2].

Em 1912, Arnstein voltou a abordar o problema, também utilizandose do método das forças, não tendo entretanto alcançado muito sucesso pelas mesmas razões anteriores. No mesmo ano, Kögler efetuou o estudo de uma ponte, obtendo algumas conclusões importantes e Lossier, baseando-se na teoria de vigas contínuas sobre apoios elásticos, apresentou também um trabalho [3].

Em 1914, Huber aplicou pela primeira vez a teoria de placas ortotrópicas para a resolução do problema. Nesta mesma época, Saliger, Frank e Knorr trabalharam no campo da pesquisa experimental e obtiveram resultados relevantes que contribuiram para o melhor conhecimento do assunto [4].

Em 1922, Thullie analisou o problema das grelhas, tomando para as transversinas uma rigidez infinita [5].

Em 1925, Petermann, adotando como incógnita os momentos nos nós das grelhas, deparou-se também com dificuldades numéricas [6].

Em 1926, Faltus simulou, pela primeira vez, o efeito de todas as transversinas do tabuleiro, representando-as por uma transversina fictícia única. Alcançou assim bons resultados para a distribuição de cargas no meio do vão [7].

Em 1927, Bleich e Melan, desprezando a rigidez torsional dos elementos da grelha, chegaram a um sistema de equações diferenciais parciais e apresentaram a solução destas [8].

Em 1928, Gennter incluiu as rijezas torsionais ao estudo anterior, mas, em virtude das dificuldades numéricas apreciáveis, não alcançou resultados 
positivos na resolução das equações diferenciais parciais [9].

Em 1930, Ostenfeld, utilizando o método dos deslocamentos e considerando cada nó como apoio indeslocável, chegou também à um sistema de equações. No mesmo ano, Krall, baseando-se na teoria de vigas sobre apoios elásticos, apresentou um trabalho sobre a repartição transversal de cargas [10].

Em 1938, Leonhardt apresentou um importante trabalho sobre grelhas apoiadas em dois bordos. Neste trabalho, foram estudados os coeficientes de distribuição transversal, desprezando-se a torção do conjunto e considerando a laje apenas como uma parcela colaborante na inércia das vigas. Em 1940, o mesmo autor estendeu o método às grelhas engastadas e contínuas, concluindo então o conhecido "Método de Leonhardt". Posteriormente, em 1950, o método foi mais uma vez aperfeiçoado por Leonhardt, com auxílio de Andrä [11].

Em 1940, Courbon desenvolveu o método dos coeficientes de distribuição transversal para grelhas constituídas por transversinas com rigidez infinita. Este método também é atribuído a Engesser, sendo assim conhecido como "Método de EngesserCourbon" [12].

Em 1946, Guyon deu continuidade ao estudo de Huber para grelhas compostas por elementos sem rigidez torsional. Com a hipótese de um elevado número de longarinas e transversinas, a grelha foi assimilada a um sistema contínuo (placa ortotrópica). Em 1950, Massonet prosseguiu com os estudos de Guyon, incluindo a rigidez à torção das vigas. Com esse aporte, concluiu o "Método dos Coeficientes de Distribuição Transversal de GuyonMassonet". Em 1955, o método foi aperfeiçoado por Rowe, que introduziu a consideração da influência do coeficiente de Poisson. Posteriormente, em 1965, o método veio a ser ampliado por Barés [13].

Em 1951, no Brasil, Ferraz apresentou um trabalho no qual fez-se uso de funções ortogonais para solução das equações diferenciais do problema de uma placa ortotrópica equivalente a uma grelha [14].

Em 1956, Homberg e Weinmeister abordaram a questão sem considerar efeitos de torção. Posteriormente, em 1962, Homberg e Trenks apresentaram um trabalho no qual os efeitos de torção foram incluídos.[15].

\subsection{MÉTODOS SEM CONSIDERAÇÃO DA TORÇÃO NAS VIGAS}

\subsubsection{Método de Engesser- Courbon [16]}

Além das hipóteses básicas relativas à Teoria das Estruturas (comportamento linear elástico, pequenos deslocamentos, seções planas, Princípio de Saint-Venant), foram consideradas ainda as abaixo descritas:

a) As longarinas são paralelas, ligadas entre si perpendicurlamente por transversinas e possuem inércia constante.

b) As transversinas estão simplesmente apoiadas nas longarinas e admite-se que estas possuem rigidez infinita à flexão, desprezando-se suas deformações em relação às deformações das longarinas.

c) Desprezam-se os efeitos de torção.

Assim, com base nestas hipóteses, as transversinas comportam-se como barras rígidas, permanecendo com seus eixos retilíneos após a deformação do conjunto.

Admitindo-se proporcionalidade entre o produto "flecha $(y) \times$ rigidez $(J)$ " 
e as reações das longarinas $(R)$, tem-se, para as cargas aplicadas nas transversinas:

$$
R_{i} \quad \alpha \quad J_{i} \cdot y_{i}=J_{i} \cdot\left(a+b \cdot x_{i}\right)
$$

A solução do problema consiste em se determinar os valores de $R_{i}$, a partir do equilíbrio do conjunto. Assim, uma vez equacionados os valores de "a" e "b", obtém-se:

$$
R_{i}=\frac{P}{n} \cdot\left[1+6 \frac{2 \cdot i-(n+1)}{n^{2}-1} \cdot \frac{e}{\varepsilon}\right]
$$

que é a expressão geral para uma reação $R_{i}$ relativa ao apoio constituído por uma longarina genérica $i$, sendo $(i=1, \ldots, n)$ e considerando-se as longarinas idênticas e igualmente espaçadas entre si.

Nas Eq. (2.1) e (2.2), tem-se:

$P$ é a carga atuante na transversina;

$n$ é o número total de longarinas;

$i$ é a longarina genérica;

$e$ é a abscissa do ponto de aplicação da carga P; longarinas.

$\varepsilon$ é o espaçamento entre as

Assim, a totalidade da carga $P$ é absorvida pelas longarinas (como se não houvesse transversinas no tabuleiro) segundo um coeficiente de repartição transversal $r_{i e}$, dado por:

$$
r_{i e}=\frac{1}{n} \cdot\left[1+6 \cdot \frac{2 \cdot i-(n+1)}{n^{2}-1} \cdot \frac{e}{\varepsilon}\right]
$$

Uma vez conhecidos os coeficientes $r_{i e}$, torna-se possível obter as solicitações e reações de apoio nas longarinas através do carregamento das linhas de influência de reação $r_{i e}$ (na transversal) e, posteriormente, do carregamento das linhas de influência das longarinas na direção longitudinal.

$\mathrm{O}$ método também permite $\mathrm{O}$ estudo de casos mais genéricos, onde as longarinas são desiguais (em inércia) e desigualmente espaçadas. Nestes casos, toma-se como origem do eixo $x \quad 0$ centroíde das seções das longarinas, afetadas de massas proporcionais às inércias correspondentes.

Os resultados obtidos por este método serão mais satisfatórios, na medida em que 0 parâmetro $\lambda$ for menor, sendo:

$$
\lambda=\frac{l}{2 L} \cdot \sqrt[4]{\frac{L}{l} \cdot \frac{n}{t} \cdot \frac{\rho_{L}}{\rho_{T}}}
$$

onde:

$L$ é o comprimento do tabuleiro;

l é a largura do tabuleiro;

$n$ é onúmero de longarinas;

$t$ é o número de transversinas;

$\rho_{L}$ é a rigidez média das longarinas $(E J)$;

$\rho_{T}$ é a rigidez média das transversinas $(E \bar{J})$.

Para casos de carga $\left(P_{h}\right)$ aplicada nas longarinas $(h)$, substitui-se a carga $\left(P_{h}\right)$ por um sistema equivalente, constituído por diversas cargas $\left(P_{h 1}, P_{h 2}\right.$, etc...) aplicadas nos pontos de cruzamento da longarina carregada $(h)$, com as transversinas que constituem a grelha. A partir desta substituição procede-se da forma descrita no caso de cargas aplicadas nas transversinas.

\subsubsection{Método de Leonhardt [17]}

Neste método, além das hipóteses básicas da Teoria das Estruturas, foram ainda admitidas as seguintes:

a) Todas as transversinas do tabuleiro são representadas por uma única 
transversina fictícia, apoiada no meio dos vãos das diversas longarinas;

b) Esta transversina fictícia é considerada como simplesmente apoiada nas longarinas;

c) Desprezam-se os efeitos de torção.

Sob ação de uma carga $P_{k}$ unitária, o conjunto se deforma, originando reações $r_{1 k}, r_{2 k}, \ldots, r_{i k}, \ldots, r_{n k}, \quad$ denominadas "coeficientes de repartição transversal", onde $r_{i k}$ é a reação correspondente à longarina "i" quando a carga unitária atua na transversina "k".

Uma vez obtidos os coeficientes $r_{i k}$, a determinação dos esforços seccionais e reações de apoio nas longarinas pode ser feita de forma idêntica à do método de EngesserCourbon. A deformabilidade do conjunto e, portanto, os valores dos coeficientes $r_{i k}$, dependem nos casos normais das seguintes grandezas:

a) $\mathrm{Da}$ relação entre inércias da transversina $(\bar{J})$ e longarinas $(J)$, expressa pelo parâmetro $\eta$, onde:

$$
\eta=\frac{\bar{J}}{J}
$$

b) Da relação entre 0 afastamento recíproco das longarinas $(\varepsilon)$ e o vão $(L)$, expressa pelo parâmetro $\lambda$, onde:

$$
\lambda=\frac{\varepsilon}{L}
$$

Assim, os coeficientes de repartição transversal serão função do grau de rigidez da estrutura, expresso pelo parâmetro $\zeta$, onde:

$$
\zeta=\frac{\eta}{(2 \cdot \lambda)^{3}}=\frac{\bar{J}}{J} \cdot\left(\frac{L}{2 \cdot \varepsilon}\right)^{3}
$$

Tomando-se $\zeta$ como parâmetro de entrada, pode-se obter os coeficientes de repartição transversal tabelados para diversos casos [17], inclusive aqueles com longarinas externas com rigidez diferente das internas. Podem ainda ser analisados casos especiais com diferentes tipos de vinculação nas longarinas.

\subsection{MÉTODOS QUE CONSIDERAM A RIGIDEZ À TORÇÃO DAS VIGAS}

\subsubsection{Método de Guyon-Massonet $[16,17]$}

Este método baseia-se na teoria geral das lajes ortotrópicas, na qual se admitem as seguintes hipóteses básicas:

a) A espessura da placa é constante e pequena em relação às demais dimensões;

b) As deformações são puramente elásticas, obedecem a lei de Hooke e os deslocamentos são pequenos em relação à espessura da laje;

c) Pontos alinhados segundo uma normal à superfície média da laje indeformada encontram-se também linearmente dispostos em uma normal à superfície média na configuração deformada;

d) Pontos situados na superfície média da laje deslocam-se somente normalmente à mesma;

e) Em relação ao material, admite-se que as propriedades elásticas sejam constantes, podendo ser diferentes nas duas direções ortogonais.

O estudo do problema foi desenvolvido, a partir destas hipóteses de comportamento da placa ortotrópica, baseando-se ainda nas premissas abaixo enunciadas:

a) O tabuleiro como um todo, composto por laje, longarinas e 
transversinas é substituído por uma placa ortotrópica equivalente. Tal associação se faz admitindo-se que os espaçamentos entre longarinas e transversinas são suficientemente pequenos para que se possa assimilar o tabuleiro a um sistema estrutural contínuo (placa);

b) A distribuição de qualquer carregamento no sistema equivalente é aproximada por meio da expressão:

$$
p(x)=p \cdot \operatorname{sen} \frac{\pi \cdot x}{L}
$$

Esta expressão define um carregamento senoidal aplicado em uma faixa genérica, situada na direção paralela ao eixo longitudinal do tabuleiro.

Considerando-se o exposto, o funcionamento estático do tabuleiro passa a ser então representado pela equação diferencial dada pela Eq. (2.9):

$\rho_{x} \frac{\partial^{4} w}{\partial x^{4}}+2 \varphi \sqrt{\rho_{x} \rho_{y}} \frac{\partial^{4} w}{\partial x^{2} \partial y^{2}}+\rho_{y} \frac{\partial^{4} w}{\partial y^{4}}=p(x, y)$

sendo:

Rigidez à flexão das longarinas

$$
\rho_{x}=\frac{E J}{l_{X}}
$$

Rigidez à flexão das transversinas

$$
\rho_{y}=\frac{E \bar{J}}{l_{y}}
$$

Parâmetro de torção

$$
\varphi=\frac{\rho_{x}+\rho_{y}}{2 \sqrt{\rho_{x} \rho_{y}}}
$$

Para o cálculo exato seria necessário solucionar a Eq. (2.9), satisfazendo as condições de contorno correspondentes. Guyon e Massonet conduziram a solução do problema de forma a obter uma série de tabelas e gráficos, nos quais podem ser encontrados os valores dos índices de repartição transversal $\chi_{\varphi}$, que dependem fundamentalmente dos seguintes parâmetros:

i) Do coeficiente de travejamento $\theta$, onde:

$$
\theta=\frac{b}{L} \cdot \sqrt[4]{\frac{\rho_{x}}{\rho_{y}}}
$$

sendo:

equivalente;

$b$ é a semi-largura da placa

$L$ é o comprimento da placa equivalente;

$\rho_{x}$ e $\rho_{y}$ são os parâmetros já definidos nas Eq. (2.10) e (2.11), respectivamente.

ii) Do parâmetro de torção $\varphi$ definido na Eq. (2.12);

iii) Da posição da carga, definida por sua excentricidade (fração da semilargura);

iv) Da posição da viga que se quer obter o índice $\chi_{\varphi}$ (fração da semilargura).

Uma vez obtidos os índices de repartição transversal, o estudo das longarinas pode ser realizado através do carregamento das direções transversal e longitudinal do tabuleiro.

\subsubsection{Método de Homberg - Trenks [15]}

O método baseia-se na teoria das grelhas e considera a rigidez torsional somente das longarinas, além da rigidez à flexão das transversinas e longarinas. A essência do método baseia-se na ortogonalização dos hiperestáticos. 
Uma grelha simplesmente apoiada com "m" longarinas e "t" transversinas é $2 t(m-1)$ vezes hiperestática. Através da ortogonalização dos hiperestáticos, a matriz $2 t(m-1)$ transforma-se em "t" matrizes independentes, cada uma associada a 2(m-1) equações e incógnitas.

Nos casos estudados (número ilimitado de longarinas) a ortogonalização é possível com grupos de cargas e de momentos, sendo necessário que as longarinas possuam inércia à flexão $J$ e à torção $\bar{J}$ constantes, e que as transversinas sejam idênticas e igualmente espaçadas entre si.

Forma-se o sistema principal estaticamente determinado, seccionandose as longarinas em (m-1) pontos. Em cada seção são aplicados os elementos dos grupos de carga e de momentos $\alpha_{h(n)}$, que são regidos pela seguinte lei:

$\alpha_{h(n)}=\alpha_{(n)}^{0} \operatorname{sen} \frac{n \pi x_{h}}{L}, \quad 0 \leq x_{h} \leq L$

onde:

$h=[1,2, \ldots, t] \quad$ abscissas de uma transversina; da série;

$$
n=[1,2, \ldots, t] \text { número de termos }
$$

$L$ é o vão das longarinas.

Os resultados deste trabalho foram apresentados na forma de tabelas que permitem sua utilização a partir do conhecimento dos seguintes parâmetros de entrada:

Rigidez à flexão da grelha:

$$
Z=\left(\frac{L}{2 a}\right)^{3} \cdot \frac{\bar{J}}{J}
$$

Rigidez à torção da grelha:

$$
Z_{T}=\frac{L}{8 a} \cdot \frac{E \bar{J}}{G J_{T}}
$$

sendo:

$L$ é o vão das longarinas;

$a$ é o espaçamento entre longarinas;

$\mathrm{J}$ é a inércia à flexão das longarinas;

$\bar{J}$ é a inércia à flexão das transversinas;

$J_{T}$ é a inércia à torção das longarinas.

As tabelas são disponíveis para um número infinito de longarinas e valores de $Z$, compreendidos entre 0 e $\infty$.

\section{MÉTODOS COMPUTACIONAIS}

\subsection{DESCRIÇÃO SINTÉTICA DOS MÉTODOS COMPUTACIO-NAIS}

\subsubsection{Evolução: Dos Métodos Clássicos ao Método dos Elementos Finitos}

Diversos métodos analíticos e numéricos aproximados, desenvolvidos antes da era computacional, vieram a ser posteriormente adaptados para serem utilizados em computadores. Este é o caso do Método das Diferenças Finitas. Métodos clássicos, como o dos Mínimos Quadrados e o Método de Ritz, também o foram. Em contraste com estes métodos anteriormente citados, o Método dos Elementos Finitos (MEF) é essencialmente um produto da era dos computadores digitais [18]. Também, opostamente àqueles métodos, o $\mathrm{MEF}$ pode ser programado para abordar problemas extremamente complexos, tais como não-linearidade física e geométrica, condições de contorno intrincadas, etc. Assim, em função da extensa aplicabilidade deste método e considerando-se ainda sua utilização neste trabalho, apresenta-se uma descrição sucinta do mesmo. 


\subsubsection{Descrição Sucinta do Método dos Elementos Finitos}

Do ponto de vista matemático, o Método dos Elementos Finitos é uma técnica utilizada para a obtenção de soluções aproximadas de problemas regidos por uma ou mais equações diferenciais. As funções incógnitas são aproximadas por funções de interpolação e, com isso, pode-se obter um sistema de equações algébricas, capaz de representar uma aproximação das equações diferenciais originais.

A principal característica do MEF consiste no fato de que ele se baseia no estudo de um elemento finito, a partir do qual todo o domínio do problema pode ser representado.

No caso de problemas ligados à Análise Estrutural, as equações diferenciais a serem aproximadas podem ser as de equilíbrio ou as de compatibilidade.

O comportamento de um elemento finito pode ser estudado a partir dos príncipios fundamentais derivados do conceito de energia potencial. Este estudo pode ser realizado através de duas vias distintas [19]:

- Aplicação do princípio da energia potencial total estacionária: Neste caso os campos de deslocamentos são aproximados por funções de interpolação e são obtidas equações de equilíbrio aproximadas. Esta forma corresponde ao método da rigidez (ou dos deslocamentos);

- Aplicação do princípio da energia potencial total complementar estacionária: Neste caso as tensões são aproximadas através de funções de interpolação, obtendo-se então equações de compatibilidade aproximadas. Esta forma corresponde ao método da flexibilidade (ou das forças).
É interessante observar que no modelo de equilíbrio as soluções aproximadas indicam um comportamento mais rígido que o real. Ao contrário, no modelo de compatibilidade as aproximações fornecem resultados mais flexíveis [20].

Na prática a primeira forma tem prevalecido, já que no método da rigidez deve-se empregar como estrutura básica, um modelo cinematicamente determinado, cuja definição pode ser automatizada, por ser única. No método da flexibilidade a estrutura básica deve ser estaticamente determinada, havendo diversas possibilidades para sua formação [21].

A aplicação do MEF pode ser subdividida em duas etapas fundamentais:

- Obtêm-se as relações forçasdeslocamentos para um elemento finito (equações de equilíbrio aproximadas). Estas relações são expressas pela transformação linear.

$$
F^{e}=K^{e} U^{e}
$$

onde:

$F^{e}$ é o vetor de forças nodais do elemento;

$K^{e}$ é a matriz da rigidez do elemento;

$U^{e}$ é o vetor de deslocamentos nodais do elemento.

- Associam-se os elementos constituintes do sistema estrutural e forma-se o sistema de equações de equilíbrio relativo à estrutura. Analogamente à relação para um elemento, tem-se para a estrutura:

$$
F=K U
$$

onde: 
$F$ é o vetor de forças nodais da estrutura;

$K$ é a matriz de rigidez da estrutura;

$\boldsymbol{U}$ é o vetor de deslocamentos nodais da estrutura.

Em geral, a matriz de rigidez de um elemento é definida por [18, 22, 23]:

$$
K^{e}=\int_{v o l} B^{t} D B d v o l
$$

onde:

$B$ é a matriz das relações deformação-deslocamento;

$D$ é a matriz das relações constitutivas.

Há, porém, outra forma de se obter a matriz de rigidez, na qual se emprega o primeiro teorema de Castigliano. Uma vez assumidas as aproximações para os deslocamentos, a energia de deformação de um elemento fica completamente definida. De acordo com o primeiro teorema de Castigliano, sabe-se que:

$$
F_{i}=\frac{\partial U}{\partial \delta_{i}}
$$

onde:

$F_{i}$ e $\delta_{i}$ são, respectivamente, a força nodal e o deslocamento nodal na direção do i-ésimo grau de liberdade;

$U$ é a energia de deformação.

De acordo com as hipóteses de comportamento linear elástico, a energia de deformação assume uma forma quadrática em relação aos deslocamentos nodais. Sendo assim, a Eq. (2.20) é uma função linear dos deslocamentos, e pode ser expressa por:

$$
F_{i}=\frac{\partial^{2} \boldsymbol{U}}{\partial \delta_{i} \partial \delta_{1}} \delta_{1}+\frac{\partial^{2} \boldsymbol{U}}{\partial \delta_{i} \partial \delta_{2}} \delta_{2}+\ldots .+\frac{\partial^{2} \boldsymbol{U}}{\partial \delta_{i} \partial \delta_{n}} \delta_{n}
$$

onde:

$$
\delta_{1}, \delta_{2}, \ldots, \delta_{n} \text { são os "n" graus de }
$$
liberdade do elemento.

Para todos os graus de liberdade, a relação completa é expressa pela transformação linear (2.17).

Observando-se a Eq. (2.21), pode-se concluir que um termo genérico da matriz $K^{e}$ é expresso por:

$$
k_{i j}=\frac{\partial^{2} \boldsymbol{U}}{\partial \delta_{i} \partial \delta_{j}}
$$

Assim, uma vez obtidos estes termos, fica definida a relação entre forças e deslocamentos, concluindo-se a primeira etapa de aplicação do MEF.

A segunda etapa consiste basicamente num problema de transformação de coordenadas. Para que as contribuições de cada elemento possam ser adicionadas na matriz global da estrutura, é necessário que os graus de liberdade estejam referidos a um mesmo sistema de eixos (sistema global). Isto se obtém através da transformação a seguir indicada [24]:

$$
K^{g}=R^{t} K^{e} R
$$

onde:

$K^{g}$ é a matriz de rigidez do elemento em relação aos eixos globais

$R$ é a matriz de rotação do elemento, dependente de sua posição em relação ao sistema global de eixos.

\section{CONCLUSÕES}

Ao padrão de análise dos métodos aqui apresentados podem ser associadas três restrições básicas:

- A obtenção de esforços, tensões e deslocamentos demanda geralmente 
três etapas distintas de cálculo (superestrutura, mesoestrutura e infraestrutura) acarretando em considerável acréscimo de tempo e trabalho para análise e correlação de resultados;

- As simplificações impostas, quer aos sistemas estruturais, quer aos modelos matemáticos de análise utilizados (Engesser-Courbon, Homberg, etc), conduzem a resultados pouco satisfatórios nos casos gerais (casos de eixos curvos, não parale-lismo das longarinas, esconsidade acentuada, etc).

Esta forma convencional e sequencial de análise, com suas consequentes restrições, deve -se basicamente aos seguintes motivos práticos:

- Em geral, a análise computacional dos efeitos de protensão nos programas comerciais de estruturas reticuladas não é automática ou, quando é, não atende adequadamente as necessidades dos casos práticos;

- O mesmo ocorre com relação à análise dos efeitos de carga móvel em estruturas espaciais, que exigem a integração de superfícies de influência.

Para que se alcançe um elevado nível de automatização da análise estrutural deste tipo de obra, deve-se desenvolver uma metodologia que disponha dos seguintes recursos:

- Aplicação do conceito de carga equivalente a um elemento de pórtico plano para transformar os efeitos da protensão em carregamento externo;

- Um programa para análise dos efeitos da protensão, com conside-ração das perdas imediatas e lentas. A partir da distribuição de tensões ao longo dos cabos são calculadas automaticamente as cargas equivalentes de protensão, de acordo com a formulação proposta;

- Um modelo estrutural para transformação do tabuleiro (lajes + vigas) numa grelha equivalente onde são consideradas as ligações excêntricas entre as vigas e o plano da laje.

- Um elemento desenvolvido especificamente para simular as diferentes formas de ligação, normalmente necessárias em uma modelagem mais refinada (aparelhos de apoio em neoprene fretado, lajes de continuidade, juntas e excentricidades de pontos nodais);

- Um programa para análise estática linear de estruturas reticuladas espaciais, baseado no método da rigidez, no qual foram implementados todos os algoritmos necessários ao processamento e análise de estruturas protendidas, modeladas e carregadas com base nos desenvolvimentos descritos nos quatro pontos anteriores.

\section{REFERÊNCIAS BIBLIOGRÁFICAS}

[1] SILVEIRA, V. L. C. "Análise Crítica da Distribuição Transversal em Tabuleiros de Pontes Simplesmente Apoiados" Tese M. Sc. PUC, Rio de Janeiro, 1976.

[2] ZSCHETZSCHE, A. "Theorie Lastvertheilender Querverbände. Zeitschrift des Oesterr" Ingenieurund Architekten-Vereines, 1893, p.553.

[3] LOSSIER, H "Étude de la Solidarité des Pieces de Pont", Le Genie Civil, 1912, p. 336.

[4] F.KNORR, "Untersuchungen úber die Last - Verteilung von Querträgern bel einer Balkenbrücke aus Eisenbeton. Arm". Beton, 1919, p. 299.

[5] M. THULLIE. "Die Duckvertei lung auf die. einzelnen Träger der 
Betonbrúcke", Beton u. Eisen", 1922, p. 67.

[6] PETERMANN. "Über Lastverteilende Virkung Durchgehender Querverbindungen", Bautechn, 1925, p. 518.

[7] F.FALTUS: "Lastverteilende Querverbindungen", Bauing, 1927, p. 853

[8] BLEIC-MELAN. "die Gewöhnlichen und partiellen Differenzengleichungen der Baustatik", 1927, Julius Springer.

[9] R.GENNTER, "Der Eisenbetonträgerrost, Beton u. Eisen 1928, p. 411.

"Lastverteilende

CHRIST.,OSTENFELD,

Querverbindungen.", Kopenhagen, 1930, jul. Gjellerup

[11] F. LEONHARDT- W.ANDRÁ, "Die Vereinfachte Trägerrostberechnung, Stuttgart", 1950, Julius Hoffmann.

[12] J.COURBON, "Calcul des Ponts à Putres Multiples Solidarisees par des Entretoises", kAnnales de Ponts eet Chaussées, Novembro Dezembro, 1940.

[13] BARÈS, R. ; MASSONET, C. "Le Calcul des Grillages de Poutres et Dalles Orthotropes", Dunod, Paris, 1966.

[14] FERRAZ, J. C. F., "Contribuição ao Estudo de Grelhas, Rio de Janeiro, 1950.

[15] HOMBERG-TRENKS, Drehsteife Kreuzwerke. Ein Handbuch fur den Brückenbau.

Berlin/Göttingen/Heidelberg:

Springer 1966.

[16] MARTIN, F. J. "Cálculo de Tabuleiros de Pontes", Ciência e Tecnologia, Rio de Janeiro, 1981.

[17] ROWE, R. E., "Concrete Bridge Desing", Applied Science Publishers Ltd., London, 1972.

[18] DESAI, C. S.; ABEL, J. F. "Introduction to the Finite Element
Method", East-West Press, New Delhi, 1972.

[19] PRZEMIENIECKI, J. S. "Theory of Matrix Structural Analysis", Dover publications, New York, 1985.

[20] FERRANTE, A. J., "Método dos Elementos Finitos", DIVEN PETROBRÁS, Rio de Janeiro, 1987.

[21] GERE, J. M.; WEAVER, W. J. "Análise de Estruturas Reticuladas", Editora Guanabara Dois, Rio de Janeiro, 198.1

[22] ZIENKIEWICZ,O.C., "The Finite Element Method", McGraw-Hill, London, 1982.

[23] BATHE, K.J., "Finite Element Procedures in Engineering Analysis", $\quad$ Prentice-Hall, Inc., Englowood Cliffs, New Jersey, 1982.

[24] GERE,, J.M., WEAVER W.J., "Matrix Algebra for Engineers", D.Van Nostranol Company, New York, 1965. 